\title{
Survival Effect of Supportive Care Services for Turkish Patients with Metastatic Gastric Cancer
}

\author{
Esat Namal ${ }^{1}$, Candas Ercetin ${ }^{2 *}$, Merve Tokocin ${ }^{2}, Z_{\text {Zafer Akcali }}{ }^{3}$, Hakan Yigitbas ${ }^{2}$, \\ Erkan Yavuz ${ }^{2}$, Fatih Celebi ${ }^{2}$, Tolga Totoz ${ }^{4}$, Ozgul Pamukcu $^{5}$, Emel Saglam ${ }^{6}$
}

\begin{abstract}
Background: Gastric cancer is the second most common cause of cancer- related deaths worldwide and ranks $11^{\text {th }}$ or $14^{\text {th }}$ among all deaths. Patients with advanced disease require supportive care along with the medical and/ or surgical treatment. $\underline{\text { Aim: }}$ : To assess the need for palliative care for patients with advanced tumours along with standard clinical therapy. Materials and Methods: Eighty-four patients with metastatic (stage 4) gastric cancer, including both patients who had received surgical treatment or not, were followed up in Bagcilar Training and Research Hospital, Division of Medical Oncology between 2011 and 2014. They were categorised as supportive care (-) (Group 1, $n=37)$ and (+) groups (Group 2, $n=47)$ and evaluated retrospectively. Results: Demographic

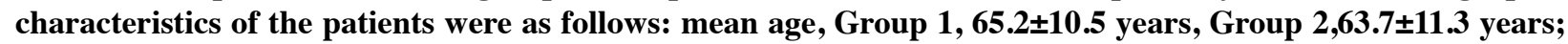
male/female ratio, Group 1, 21/16, Group 2, 28/19; distribution of Eastern Cooperative Oncology Group (ECOG) performance scores of 0 and 1, Group 1, ECOG $0(n=9)$ and $1(n=14)$, Group 2, ECOG 0 (34) and 1 $(n=13)(p<0.0001)$; patients receiving second-line, Group $1(n=7)$ and Group $2(n=22)(p<0.008)$ or third - line chemotherapy,Group $2(n=6)(p<0.02)$; mortality rates, Group 1, $(n=28 ; 75.6 \%)$ and Group $2(n=30 ; 63.8 \%)$; progression-free survival (PFS) rates, Group 1, 17.4 \pm 6 weeks, Group 2, 28.3 \pm 16.2 weeks; statistically significant overall survival rates, Group 1, 20.8 \pm 8.2 weeks and Group 2, $28.3 \pm 162$ weeks $(p<0.01)$. Conclusions: The supportive care team (medical oncologist, general surgeon, internal medicine specialist, algologist, psychiatrist and radiologist) can play a role in the treatment of metastatic gastric tumours, with improvements shown in terms of the performance status of cases, eligibility of patients to be on chemotherapy programmes for longer duration and overall survival rates in Turkey.
\end{abstract}

Keywords: Metastatic gastric cancer - supportive care team - chemotherapy - Turkey

Asian Pac J Cancer Prev, 16 (3), 1213-1217

\section{Introduction}

Despite advances in the treatment of gastric cancer, it still remains to be the second leading cause of cancer deaths in the world. As gastric cancer is often diagnosed at an advanced stage, systemic chemotherapy is the mainstay of the treatment. However, no standard palliative chemotherapy regimen has been accepted for the patients with metastatic gastric cancer. Palliative chemotherapy including fluoropyrimidine, platin-based chemotherapies, docetaxel and epirubicin prolongs survival and improves the quality of life to a greater extent than the best supportive care (Bilici, 2014).

Gastric cancers with metastases or recurrences have very poor prognosis; but chemotherapy can improve survival and possibly provide significant palliation of symptoms. Despite recently reported benefits of chemotherapy, the 5-year survival rate for these groups has remained at 5-20\% (Bang et al., 2010; Van Cutsem et al., 2006).

Performance status is a widely accepted parameter that predicts response to chemotherapy and survival and it is associated with many factors such as age, previous chemotherapy and comorbid diseases (Lavin et al., 1982; Janunger et al., 2001; Wilson et al., 2005). In advanced gastric cancer, patients are likely to suffer from poor general condition due to anorexia and weight loss, often as a consequence of peritoneal carcinomatosis (Cunningham et al., 1987; Hsu et al., 1997).

Managing symptoms and effective communication with patients are essential aspects for providing highquality cancer care, especially among patients with advanced cancer (Walling et al., 2012).

In particular, the patients with advanced cancer have to face many problems during the progression of the disease such as pain, fatigue, energy depletion, and loss of

${ }^{1}$ Department of Medical Oncology, Florence Nightingale Hospital, ${ }^{2}$ Department of General Surgery, ${ }^{4}$ Department of Anesthesiology, ${ }^{6}$ Department of Internal Medicine, Bagcilar Training and Research Hospital, ${ }^{5}$ Department of Internal Medicine, Sisli Etfal Training and Research Hospital, Istanbul, ${ }^{3}$ Department of Medical Oncology, Ufuk University, Dr. Ridvan Ege Hospital, Ankara, Turkey *For correspondence: ercetin@istanbul.edu.tr 
appetite, along with physical symptoms of cancer itself. In addition, cancer patients have a variety of psychological symptoms, suggestive of anxiety, depression, and sleep disorder (Park et al., 2012). The prevalence of physical and psychological symptoms are varied according to the reported research by $35-96 \%$ for pain, 3-77\% for depression, $13-79 \%$ for anxiety, 32-90\% for fatigue, 10$70 \%$ for dyspnea, and 9-69\% for sleep disorders (Solano et al., 2006).

In this study, we aimed to point out that palliative care need to be administered to the patients with advanced tumours along with the medical and/or surgical treatments.

\section{Materials and Methods}

The patients with metastatic (stage 4) gastric cancer - including both patients who had surgical treatment or not (in our general surgery clinic or in another center) who were followed up in Bagcilar Training and Research Hospital, Division of Medical Oncology in between 2011 and 2014 were evaluated retrospectively.

Patients were staged based on the combination of data obtained from endoscopic examinations, thoracic and abdominal CT (computed tomography) and PET (positron emission tomography) or eventually bone scans if clinically indicated. The inclusion criteria were as follows: histologically confirmed gastric cancer, no prior chemotherapy or radiotherapy except adjuvant treatment, presence of metastatic disease and availability of clinical data at the initiation of therapy and follow-up. Eastern Cooperative Oncology Group performance status (ECOGPS) was evaluated according to the Eastern Cooperative Oncology Group criteria.

Our supportive care team was set up with the leadership of medical oncology and comprised of the professional academicians from the fields of general surgery, algology, psychiatry, emergency medicine, internal medicine, radiology, nuclear medicine and radiation medicine. Communication and coordination between the disciplines were organised by medical oncology. Eighty-four cases on follow- up with stage 4 gastric cancer were included into the study and categorised into groups who received (Group 2, n=47) or did not receive (Group 1, n=37) supportive care.

Medical files of the patients were examined thoroughly and the following data were recorded: demographic characteristics (age, sex), localization of the tumour, information about the previous operation (if any), spread of the disease, chemotherapy regimens, histopathological evaluation, ECOG-PS, progression- free survival (PFS), overall survival (OS), complications and mortality.

A physical examination including evaluation of weight, performance status and laboratory tests was performed before each subsequent cycle.

Statistical Analysis: Statistical analyses of this study was made by using NCSS (Number Cruncher Statistical System) 2007 Statistical Software (Utah, USA) package programme. As normality test, Kolmogorov - Smirnov test was used. Besides definitive statistical methods for the evaluation of the data (mean, standard deviation, frequency distrubutions) and also dependent t test were used for the comparison of paired samples and chisquare test was used for the comparison of qualitative data. Survival curves were drawn using Kaplan Meier method and log-rank test was used for the comparison of the survival curves. Kaplan-Meier analysis was applied to assess factors affecting overall survival (OS) and progression- free survival (PFS). OS was defined as the time from the first day of the first-line chemotherapy or to death from any cause or the last follow-up visit. PFS was defined as the time from the first day of the firstline chemotherapy to disease progression. $\mathrm{p}<0.05$ was accepted as the level of statistical significance.

\section{Results}

All cases were metastatic (stage IV) gastric cancer. The median age of the patients was 67 (range 36 to 87) years with a male/female ratio of 49/35 $(n=84)$. Twentyone of these cases were referred to our medical oncology outpatient clinic following the surgical intervention in Bagcilar Training and Research Hospital, Division of General Surgery or in an external surgical clinic.

When subgroups were analysed, median ages of the patients in Groups 1 and 2 were 67 (range, 45 to 87) and 66 (range, 36 to 87 ) years, respectively. Male to female ratio was 21/16 in Group 1 and 28/19 in Group 2. Any statistically significant difference was not found between the mean ages and male/female ratios of two groups ( $\mathrm{p}=0.525$ and $\mathrm{p}=0.244$, respectively) (Table 1$)$.

The patients underwent either total (Group 1, $n=4 / 7$; Group 2, $n=10 / 14$ ) or distal subtotal (Group 1, n=3/7; Group 2, n=4/14) gastrectomies.

Eastern Cooperative Oncology Group (ECOG) performance scores were also determined as follows: Group 1, ECOG $0(n=9 ; 24.3 \%)$, ECOG 1 ( $n=14$ cases; $37.8 \%)$, ECOG $2(n=13 ; 37.8 \%)$, ECOG $3(n=1 ; 2.7 \%)$ and Group 2, ECOG $0(n=34 ; 72.3 \%)$, ECOG $1(n=13$; $27.6 \%)$. The number of patients with ECOG score of 0 $(72.34 \%)$ and $1(27.66 \%)$ in Group 2 was significantly higher than the number of patients with ECOG score 0 $(24.32 \%)$ and $1(37.84 \%)$ in Group $1(\mathrm{p}=0.0001)$ (Table 1$)$.

Seven patients (18.9\%) in Group 1 and 22 (46.8\%) patients in Group 2 received second cycle of chemotherapy with a statistically significant difference between groups $(\mathrm{p}=0.008)$. Only in Group 2, $6(12.7 \%)$ patients received third cycle of chemotherapy with a significant difference between groups $(\mathrm{p}=0.024)$ (Table 1$)$.

Histopathologically, adenocarcinomas (Group 1, n=29 and Group 2, n=36), signet cell carcinomas (Group 1, n=6; Group 2, n=8) and mucinous carcinomas (Group 1, n=2 and Group 2, n= 3) were detected (Table 1).

Any significant difference was not detected between the progression- free survival times of two groups (Group $1,17.49 \pm 6.87$ weeks and Group 2, 21.68 \pm 13.15 weeks) $(\mathrm{p}=0.082$ ) (Table 1.). Based on Kaplan Meier survival curves, there was no significant difference between the groups as for the median survival times of the groups [Group 1, 19 (range, 16.3 to 21.6 ] weeks (SE: 1,36) and Group 2, 29.8 (range, 23.2 to 36.3) weeks (SE: 3.36) with the long rank $2.63(\mathrm{p}=0.105)$ (Figure 1$)$.

The overall survival times of the patients were 
$20.89 \pm 8.26$ and $28.32 \pm 16.28$ weeks in Groups 1 and 2 , respectively. As is seen- it was statistically significantly higher in Group 2 ( $\mathrm{p}=0.013$ ) (Table 1). Based on Kaplan Meier survival curve, median survival times were 22.3 (range, 19.3 to 25.4 ) weeks (SE: 1.54) and 35.5 (range, 29 to 42 ) weeks (SE: 3.31 ) in Groups 1 and 2, respectively which was found to be significantly longer in Group 1 with a log rank value of 6.53 ( $\mathrm{p}=0.011$ ) (Figure 2).

Table 1. Age, Gender, ECOG, Chemotherapy Cure, Histopathological Examination, PFS and OS Time, Mortality of group 1 and 2

\begin{tabular}{lccl}
\hline & Group 1 & Group 2 & p value \\
\hline Age (years) & $67(45-87)$ & $66(36-87)$ & ns \\
Male / Female (n) & $21 / 16$ & $28 / 19$ & ns \\
ECOG (n) & & & \\
0 & 9 & 34 & 0.0001 \\
1 & 14 & 13 & 0.0001 \\
2 & 13 & - & \\
3 & 1 & - & \\
Chemotherapy (n) & & & \\
Second line & 7 & 22 & 0.008 \\
Third line & - & & 0.024 \\
Histopathologically (n) & & 36 & \\
Adenocarcinoma & 29 & 8 & \\
Signet cell carcinoma & 6 & 3 & \\
Mucinous carcinoma & 2 & $21.7 \pm 13.2$ & 0.08 \\
PFS (week) & $17.5 \pm 6.87$ & $28.3 \pm 16.3$ & 0.013 \\
OS (week) & $20.9 \pm 8.26$ & 30 & 0.24 \\
Mortality (n) & 28 & &
\end{tabular}

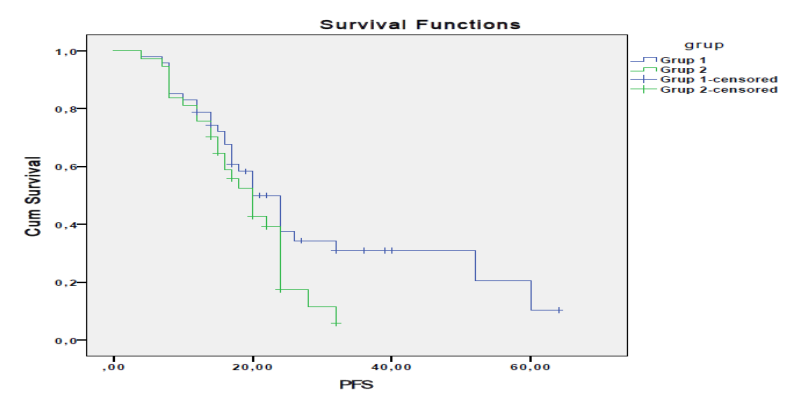

Figure 1. The Kaplan Meier Survival Curves (for PFS).There was no significant difference between the groups as the median of group 1 was 19 weeks (16.3-21.6) (SE: 1,36), and group 2 was 29,8 weeks (23.2-6.3) (SE: 3.36) with the long rank $2.63(\mathrm{p}=0.105)$

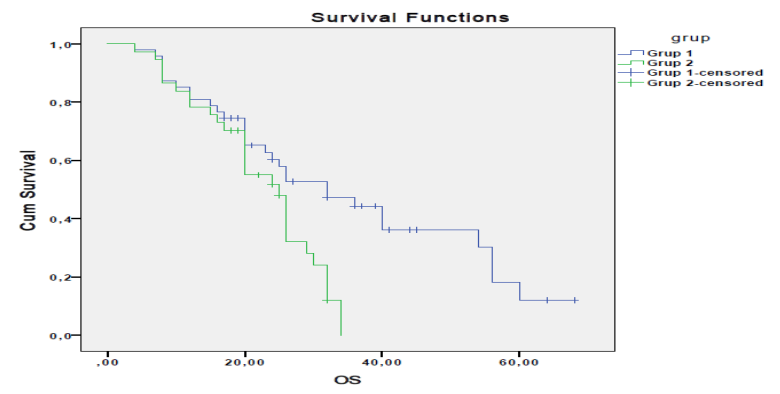

Figure 2. The Kaplan Meier Survival Curve (for OS). Medians were 22,3 weeks (19.3-25.4) (SE: 1.54) and 35.5 weeks (29-42) (SE: 3.31) in group 1 and 2 respectively; and it was found significantly longer in group 1 with log rank value of $6.53(\mathrm{p}=0.011)$
Any significant difference was not found between the mortality rates of the two groups (Group 1, $\mathrm{n}=28 ; 75.6 \%$ and Group 2, n=30;63.8\%) $(\mathrm{p}=0.244)$ (Table 1).

\section{Discussion}

As the number of cancer patients increases, new treatments methods are developed, symptoms are controlled, and psychological and physiological quality is improved; however, patients are directed to home care due to the lack of staff, accommodations and economic difficulties (Ugur et al., 2014).

Gastric cancer is the second most common cause of cancer deaths worldwide. Although the overall incidence and mortality of this disease have dramatically declined over the last few decades, it still remains a major health problem (Hwang et al., 2011; Pasini et al., 2011). Radical gastrectomy is the only curative treatment of gastric cancer, but recurrence is common, being detected in approximately $60 \%$ of the patients (Bilici, 2014). In addition, gastric cancer is often diagnosed at an advanced stage, excepting Japan and Korea, where screening for gastric cancer is widely performed. For these patients, systemic chemotherapy is the mainstay of treatment (Jeung et al., 2007; Glimelius et al., 1997; Siegel et al., 2013; Wagner et al., 2010). Although various chemotherapeutic agents, either alone or in combination, have been studied since 1970, the median survival of patients with a metastatic disease remains between 6 and 9 months (Shin et al., 2004; Greenlee et al., 2000).

Chemotherapy-based comprehensive treatment is adopted for the treatment of late gastric cancer, but the curative effect is not ideal. In recent years, in order to improve the efficacy and the survival time of the patients, targeted drugs combined with chemotherapy regimens come into being (Xu, 2014).

Gastric cancer is one of common malignant tumors, with the ORR of the first-line chemotherapy less than $60 \%$ and the median OS being 6.0 10.0 months ( Tomasello et al., 2014). At present, there were no ideal drugs or combined therapies for the treatment of gastric cancer. And chemotherapy was regarded as the main method for treating late gastric cancer (Liu et al., 2014). However, it has limitation in clinical application because patients can't tolerate its toxic and side effects (Xu, 2014).

Patients with inoperable, recurrent or metastatic tumours are incurable and they survive only a few months with the best supportive care (BSC). In this setting, systemic chemotherapy prolongs survival and improves symptom palliation, although these benefits are to be weighed against treatment-related toxicities (Pasini et al., 2011; Al-Batran et al., 2008; Kang et al., 2010).

Old age, poor performance status, comorbidities and significant weight loss are adverse prognostic factors for gastric cancer (Andreyev et al., 1998; Trumper et al., 2006).

Supportive care is defined as the provision of the necessary services for those living with or affected by cancer to meet their physical, emotional, social, psychological, informational, spiritual and practical needs during the diagnostic, treatment, and follow-up phases, 
encompassing issues of survivorship, palliative care and bereavement (Park et al., 2012). The supportive care of patients with cancer improves patients' quality of life (Rosenbaum et al., 2004) and increases patients' survival rate (Alfano and Rowland, 2006).

Performance status is an indicator of a patient's global ability and it correlates with survival time. Preoperative performance status of ECOG 2-3 in non-curable gastric cancer patients is associated with a 1-year survival rate of $17 \%$, compared with $43 \%$ for ECOG $0-1$ patients (Maehara et al., 1993).

Taking a look at research that deals with Supportive Care or Perception of a medical team for Palliative Care, in the study which was investigated attitude toward palliative care of advanced cancer patients (European Society of Medical Oncology Taskforce on Palliative and Supportive Care (ESMO), 2003), most oncologists thought supportive care or palliative care was important. In addition, they said they were dealing with physical symptoms, such as general pain, fatigue, emesis, and cures for conditions, that is, depression, anxiety, mental distress and delirium. Among them, 74\% were satisfied with care for terminally ill patients, but $42 \%$ responded they had lack of training to accomplish these (Park et al., 2012).

The results of some studies showed that cancer survivors in Western and Eastern countries reported that the level of social support available for them is high (Cheng et al., 2013; Faghani et al., 2014).

As we aimed to evaluate the effect of supportive care to the course of disease, we have observed our patients following set up of our supportive care team. It was observed that durations of follow- up and treatment of the patients with advanced metastatic tumours have been prolonged after the supportive care team had been assigned.

In the evaluation of corresponding disciplines separately, we have found that each clinic played a role in the management of patients ie: general surgery team evaluated the suitability of the patient for operation; algology team provided palliative treatment for pain; psychiatry team provided psychological support to the patients and their relatives; emergency medicine team stabilised the patient and coordinated corresponding disciplines following the first examination of the patients in case of emergency; internal medicine team planned the supportive care of the patient such as nutrition, fluid and electrolyte distubances before or during the chemotherapy by hospitalization of the patient; radiology team facilitated the treatment selection process by staging, determining progression/regression and palliative treatment alternatives; nuclear medicine team took part in staging of the disease; radiation oncology team planned the radiotherapy processes and dieticians played a role in the regulation of nutrition and diets of the patients. It has been thought that multidisciplinary approach has exerted a positive effect on OS, PFS and quality of life of the patients.

This hypothesis is based on the circumstances that are typical for these tumours: 1- Their prognosis is generally poor. In oesophageal and pancreatic cancer, prognosis is worse than any other tumour (Berrino et al., 1999). 2- The diagnostic procedure is complex. It is crucial to establish the tumour stage reliably, since this determines the choice of treatment. 3- The treatment is demanding. Extensive surgery is usually required to offer any chances of cure (Enzinger and Mayer, 2003). This surgical treatment, especially in oesophageal cancer, carries a substantial risk of severe complications (Viklund et al., 2006) and of long-term impairment of the quality of life (Viklund et al., 2005). 4- Patient management is referred to a few reference hospitals. Since it has been proven that highvolume surgery for oesophageal and pancreatic cancer substantially improves the survival (Birkmeyer et al., 2002; Birkmeyer et al., 2003), these patients are now being referred to few reference hospitals. In view of the complex, multidisciplinary and increasingly centralised care pathway of these patients and since these patients in general often suffer considerably both from their symptoms caused by the tumour and from psychosocial problems involved in the awareness of their newly diagnosed cancer (Winterling et al., 2004), it is important to ascertain optimal care, qualified coordination and continuous support (Vinklunda et al., 2006).

In addition, it was found that chemotherapy side effects, depression, anxiety and psychological problems were often involved. Such symptoms were often worked primarily with medical specialists from other departments, and it showed that a new system distinguished from a palliative care or hospice was needed (Park et al., 2012).

The patients found the support given by the specialized oncology team which seems to be important during the entire care pathway, particularly during the follow-up after treatment. Almost all patients agreed that the supportive care given by the specialized oncology team was not only satisfactory but also highly important (Vinklunda et al., 2006).

Specialized oncology team might be of particular relevance for the patients with upper gastrointestinal cancers in view of the complexity of the diagnostic procedures, the advanced surgical treatment, reduced quality of life after surgery and the poor prognosis of these cancers.

In conclusion, thanks to the beneficial role played by the supportive care team (medical oncologist, general surgeon, internal medicine specialist, algologist, psychiatrist and radiologist) in the treatment of metastatic gastric tumours, improvements have been achieved in terms of the performance status of the cases, eligibility of patients to be on chemotherapy programmes for longer durations and overall survival rates.

\section{References}

Al-Batran SE, Hartmann JT, Probst S, et al (2008). Phase III trial in metastatic gastroesophageal adenocarcinoma with fluorouracil, leucovorin plus either oxaliplatin or cisplatin: a study of the Arbeitsgemeinschaft Internistische Onkologie. J Clin Oncol, 26, 1435-42.

Alfano CM, Rowland JH (2006). Recovery issues in cancer survivorship: a new challenge for supportive care. Cancer $J, \mathbf{1 2}, 432-43$.

Andreyev HJN, Norman AR, Oates J, Cunningham D (1998). Why do patients with weight loss have a worse outcome 
when undergoing chemotherapy for gastrointestinal malignancies? Eur J Cancer, 34, 503-9.

Bang YJ, Van Cutsem E, Feyereislova A, et al (2010). ToGA Trial Investigators: Trastuzumab in combination with chemotherapy versus chemotherapy alone for treatment of HER2-positive advanced gastric or gastro-oesophageal junction cancer (ToGA): a phase 3, open-label, randomised controlled trial. Lancet, 376, 687-97.

Bilici A (2014). Treatment options in patients with metastatic gastric cancer: Current status and future perspectives. World J Gastroenterol, 20, 3905-15.

Berrino F, Capocaccia R, Esteve J, et al (1999). Survival of cancer patients in europe: the eurocare- 2 study. international agency for research on cancer 1999.

Birkmeyer JD, Siewers AE, Finlayson EV, et al (2002). Hospital volume and surgical mortality in the United States. New England J Med, 346, 1128-37.

Birkmeyer JD, Stukel TA, Siewers AE, et al (2003). Surgeon volume and operative mortality in the United States. $N$ Engl J Med, 349, 2117-27.

Cheng H, Sit JW, Chan CW, et al (2013). Social support and quality of life among Chinese breast cancer survivors: Findings from a mixed methods study. Eur J Oncol Nurs, 17, 788-96.

Cunningham D, Hole D, Taggart DJ, et al (1987). Evaluation of the prognostic factors in gastric cancer: the effect of chemotherapy on survival. Br J Surg, 74, 715-20.

Enzinger PC, Mayer RJ (2003). Esophageal cancer. New England J Med, 349, 2241-52.

Faghani S, Rahmani A, Parizad N, et al (2014). Social support and its predictors among Iranian cancer survivors. Asian Pac J Cancer Prev, 15, 9767-71.

Glimelius B, Ekstrom K, Hoffman K, et al (1997). Randomized comparison between chemotherapy plus best supportive care with best supportive care in advanced gastric cancer. Ann Oncol, 8, 163-8.

Greenlee RT, Murray T, Bolden S, Wingo PA (2000). Cancer statistics, 2000. CA Cancer J Clin, 50, 7-33.

Hsu CH, Yeh KH, Chen LT, et al (1997). Weekly 24-h infusion of high-dose 5-fluorouracil and leucovorin in the treatment of advanced gastric cancer: an effective and low-toxic regimen for patients with poor general condition. Oncology, $\mathbf{5 4 , 2 7 5 - 8 0 . ~}$

Hwang JE, Kim HN, Kim DE, et al (2011). Prognostic significance of a systemic inflammatory response in patients receiving first-line palliative chemotherapy for recurred or metastatic gastric cancer. BMC Cancer, 11, 489.

Janunger KG, Halfstrom L, Nygen P, Glimelius B (2001). A systematic overview of chemotherapy effects in gastric cancer. Acta Oncol, 40, 309-26.

Jeung HC, Rha SY, Shin SJ, et al (2007). A phase II study of S-1 monotherapy administered for 2 weeks of a 3 -week cycle in advanced gastric cancer patients with poor performance status. Br J Cancer, 97, 458-63.

Kang Y, Ohtsu A, Van Cutsem E, et al (2010). AVAGAST: A randomized, double-blind, placebo-controlled, phase III study of first-line capecitabine and cisplatin plus bevacizumab or placebo in patients with advanced gastric cancer (AGC). Proc ASCO, 28, 4007.

Lavin PT, Bruckner HW, Plaxe SC (1982). Studies in prognostic factors relating to chemotherapy for advanced gastric cancer. Cancer, 50, 2016-23.

Liu J, Huang XE, Feng JF (2014). Further study on pemetrexed based chemotherapy in treating patients with advanced gastric cancer (AGC). Asian Pac J Cancer Prev, 15, 6587-90.

Maehara Y, Sugimachi K, Ogawa M, et al (1993). Influence of preoperative performance status on survival time of patients with advanced gastric cancer following noncurative resection. Anticancer Res, 13, 201-4.

Park SA, Chung SH, Shin EH (2012). Attitudes of nurses toward supportive care for advanced cancer Patients. Asian Pac J Cancer Prev, 13, 4953-8.

Pasini F, Fraccon AP, Manzoni G (2011). The role of chemotherapy in metastatic gastric cancer. Anticancer research, 31, 3543-54.

Rosenbaum E, Gautier H, Fobair P, et al (2004). Cancer supportive care, improving the quality of life for cancer patients. A program evaluation report. Support Care Cancer, 12, 293-301.

Shin HR, Jung KW, Won YJ, Park JG (2004). 2002 annual report of the Korea Central Cancer Registry: based on registered data from 139 hospitals. Cancer Res Treat, 36, 103-14.

Siegel R, Naishadham D, Jemal A (2013). Cancer statistics, 2013. CA Cancer J Clin, 63, 11-30.

Solano JP, Gomes B, Higginson IJ ( 2006). A comparison of symptom prevalence in far advanced cancer, AIDS, heart disease, chronic obstructive pulmonary disease and renal disease. J Pain Symptom Manage, 31, 58-69.

Tomasello G, Liguigli W, Poli R, et al (2014). Efficacy and tolerability of chemotherapy with modified dose-dense TCF regimen (TCF-dd) in locally advanced or metastatic gastric cancer: final results of a phase II trial. Gastric Cancer, 17, 711-7.

Trumper M, Ross PJ, Cunningham D, et al (2006). Efficacy and tolerability in elderly patients with advanced oesophagogastric cancer: a pooled analysis of three clinical trials. Eur J Cancer, 42, 827-34.

Ugur O, Elcigil A, Arslan D, Sonmez A (2014). Responsibilities and difficulties of caregivers of cancer patients in home care. Asian Pac J Cancer Prev, 15, 725-9.

Walling AM, Asch SM, Lorenz KA, et al (2012). The quality of supportive care among inpatients dying with advanced cancer. Support Care Cancer, 20, 2189-94.

Wagner AD, Unverzagt S, Grothe W, et al (2010). Chemotherapy for advanced gastric cancer. Cochrane Database Syst Rev, 3, 4064 .

Wilson D, Hiller L, Geh JI (2005). Review of second-line chemotherapy for advanced gastric adenocarcinoma. Clin Oncol, 17, 81-90.

Winterling J, Wasteson E, Glimelius B, et al (2004). Substantial changes in life: perceptions in patients with newly diagnosed advanced cancer and their spouses. Cancer Nursing, 27, 381-8.

Van Cutsem E, Moiseyenko VM, Tjulandin S, et al (2006). V325 study Group: Phase III study of docetaxel and cisplatin plus fluorouracil compared with cisplatin and fluorouracil as first-line therapy for advanced gastric cancer: a report of the V325 Study Group. J Clin Oncol, 24, 4991-7.

Viklund P, Lindblad M, Lagergren J (2005). Influence of surgeryrelated factors on quality of life after esophageal or cardia cancer resection. World J Surg, 29, 841-8.

Viklund P, Lindblad M, Lu M, et al (2006). Risk factors for complications after esophageal cancer resection: a prospective population-based study in Sweden. Ann Surgery, 243, 204-11.

Viklunda P, Wengstro Y, Lagergrena J (2006). Supportive care for patients with oesophageal and other upper gastrointestinal cancers: The role of a specialist nurse in the team. European J Oncol Nurs, 10, 353-63.

$\mathrm{Xu}$ CD (2014). Clinical study of nimotuzumab combined with chemotherapy in the treatment of late stage gastric cancer. Asian Pac J Cancer Prev, 15, 10273-6. 